\title{
Spectroscopic UV observations of M 1.0 class solar flare from IRIS satellite
}

\author{
Viacheslav M Sadykov ${ }^{1}$, Alexander G Kosovichev ${ }^{1,2}$, \\ Ivan N Sharykin ${ }^{3}$ and Santiago Vargas Dominguez ${ }^{4}$ \\ ${ }^{1}$ New Jersey Institute of Technology, Newark, NJ 07102, USA \\ email: vsadykov@njit.edu \\ ${ }^{2}$ NASA Ames Research Center, Moffet Field, CA 94035, USA \\ ${ }^{3}$ Space Research Institute (IKI), Moscow 117997, Russia \\ ${ }^{4}$ Observ. Astron. Univ. Nacional de Colombia, Bogotá 111321, Colombia
}

\begin{abstract}
This work presents an analysis of UV spectroscopic observations from the IRIS satellite of an M1.0 class flare occurred on 12 June 2014 in active region NOAA 12087. Our analysis of the IRIS spectra and Slit-Jaw images revealed presence of a strongly redshifted chromospheric jet before the flare. We also found strong emission of the chromospheric lines, and studied the C II 1334.5 $\AA$ line emission distribution in details. A blueshift of the Fe XXI line across the flaring region corresponds to evaporation flows of the hot chromospheric plasma with a speed of $50 \mathrm{~km} / \mathrm{s}$. Although the enhancement of the C II line integrated redshift correlates with the flare X-ray emission, we classify the evaporation as of a "gentle" type because of its long time scale and subsonic velocities. Analysis of X-ray data from the RHESSI satellite showed that both, an injection of accelerated particles and a heat flux from the energy release site can explain the energetics of the observed event.
\end{abstract}

Keywords. Sun: activity, flares, magnetic fields, atmosphere, UV radiation

\section{Introduction}

Spectroscopic observations represent a powerful instrument to study solar flares (Milligan 2015). For the processes associated with plasma flows (like jets and chromospheric evaporations (Brosius \& Phillips 2004; Milligan et al. 2006; Doshchek et al. 2013)) the spectroscopic observations are essentially important. The recent NASA's Interface Region Imaging Spectrograph (IRIS) mission (De Pontieu et al. 2014) allows us to observe several strong UV lines forming in the upper chromosphere and transition region (e.g. MgIIh\&k, SiIV and CII lines) and in the high-temperature coronal plasma (Fe XXI line). High spectral $(26-53 \mathrm{~m} \AA)$, spatial $(0.33 \mathrm{arcsec})$, and temporal $(\approx 2 \mathrm{sec})$ resolutions make this telescope a unique instrument for the spectroscopic observations.

The M1.0 class flare of 12 June, 2014 was of our particular interest because of the simultaneous observations by the IRIS satellite and New Solar Telescope (NST, Goode et al. 2010). For the NST observational details, we refer to Sadykov et al. (2014) and Kumar et al. (2015). We have also used data from the GOES (Bornmann et al. 1996) and RHESSI (Lin et al. 2002), and from Solar Dynamic Observatory (SDO) Helioseismic and Magnetic Imager (HMI) (Scherrer et al. 2012) and Atmospheric Imaging Assembly (AIA) (Lemen et al. 2012) instruments. 

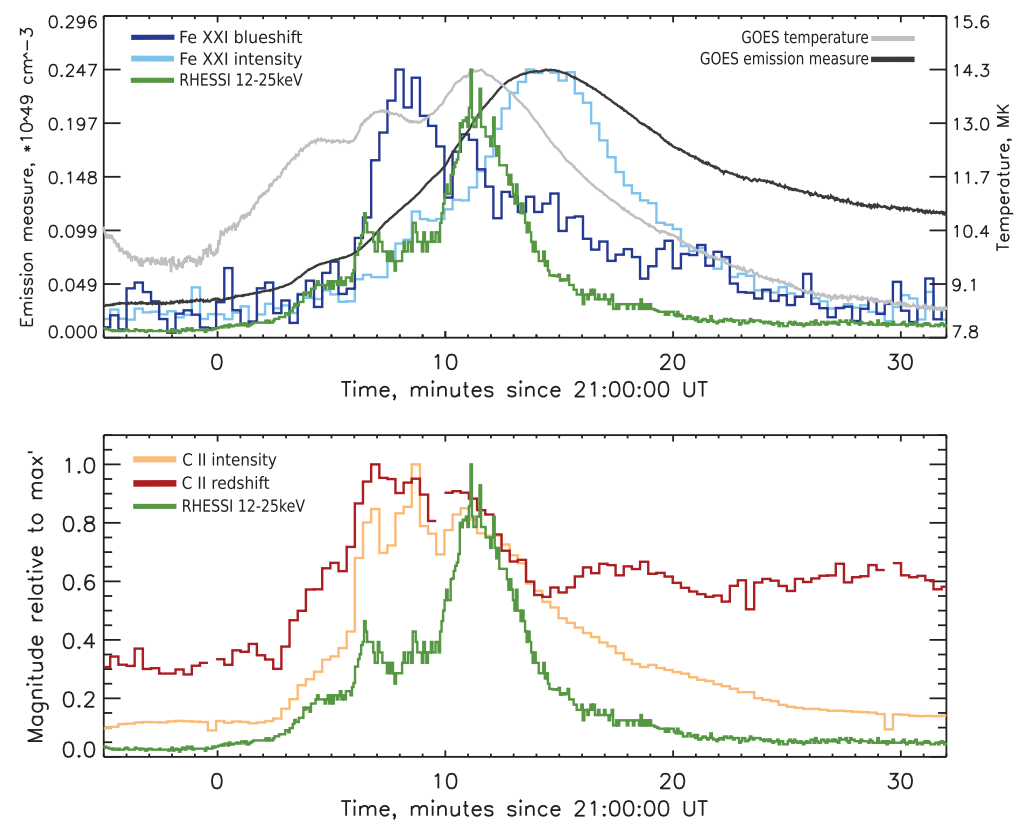

Figure 1. Upper panel: the time curves of the integrated Fe XXI $1354.1 \AA$ line blueshift (dark blue) and its peak intensity (light blue). Additionally the RHESSI $12-25 \mathrm{keV}$ flux (green), the GOES temperature (gray) and the GOES emission measure (black) are shown. Lower panel: the time curves of the integrated C II $1334.5 \AA$ line redshift (red) and its peak intensity (orange). Additionally the RHESSI $12-25 \mathrm{keV}$ flux (green) is shown.

\section{Observational overview}

The studied flare event was produced in the region located approximately 50 deg southeast from the solar disc center. The flare X-ray flux peak occurred near 21:12 UT. The RHESSI 12-25 keV flux is presented in Figure 1. The hard X-ray flux from the flare was not high; however, it was sufficient to reconstruct the $12-25 \mathrm{keV}$ sources.

IRIS observed in the coarse-raster mode ( 8 slit positions covering the region of interest in a cyclic order), which allowed us to reconstruct the spatial structure of spectral line properties during the flare. For the cold transition region lines (Si IV $1403 \AA$, C II 1334/1335 $\AA$ and Mg II k\&h $2796 \& 2803 \AA$ ) we performed the following analysis steps (see Sadykov et al. (2015) for details): 1) determine the intensity maximum (the most intense point of the line); 2) calculate the Doppler shift estimate as $\langle\lambda\rangle-\lambda_{\text {ref }}=$ $\int \lambda I d \lambda / \int I d \lambda-\lambda_{\text {ref }}$, where $\lambda_{\text {ref }}$ is the reference wavelength of the line; 3) estimate the line half-width as $\sqrt{\left\langle(\lambda-\bar{\lambda})^{2}\right\rangle}=\sqrt{\int \lambda^{2} I d \lambda / \int I d \lambda-\bar{\lambda}^{2}}$. In addition, IRIS detected the forbidden transition Fe XXI 1354.1 $\AA$ line, which appeared during the flare in the O I spectral window, and represents the dynamics of the hot $10 \mathrm{MK}$ plasma. Because this line is blended by the narrow CI1354.29 $\AA$ line, we performed a two-Gaussian fitting for the spectra, and derived all the parameters from these fits.

Our findings are the following:

- A strongly redshifted jet (collimated plasma flow) is detected in the vicinity of the magnetic neutral line prior the flare. The jet crosses the four rightmost slit positions, and a detailed study of its spectra revealed an additional redshifted (for $\sim 100 \mathrm{~km} / \mathrm{s}$ ) peak of Mg II and C II lines corresponding to this jet. Possibly, we observe the plasma flow from the reconnection region. 


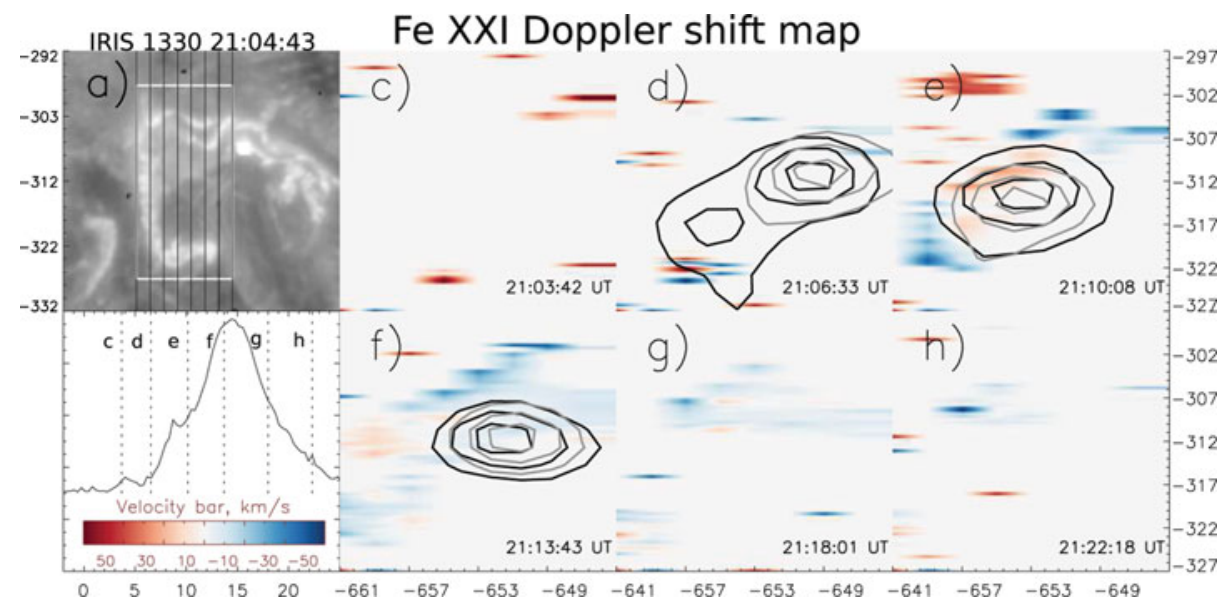

Figure 2. a) The IRIS $1330 \AA$ SJ image. The slit positions are marked by black lines, and the white rectangle represents the region for which the Fe XXI Doppler shift maps are constructed. b) The FeXXI integrated intensity time curve. The vertical lines correspond to the time moments for which the Doppler shift maps in panels c)-h) are plotted. Additionally, the RHESSI 6-12 keV (gray) and $12-25 \mathrm{keV}$ (black) sources are plotted for the corresponding times (20:04:00 UT-20:06:00 UT for the panel d), 20:07:50 UT-20:09:50 UT for e), and 20:12:00 UT-20:14:00 UT for $\mathrm{f})$ ). The Doppler shift scale is shown on the panel b).

- A sharp increase of emission of the "cold" UV lines is observed during the impulsive phase of the flare. In particular, the C II 1334.5 $\AA$ line emission is localized in small points (about $\sim 1$ arcsec in diameter) distributed along the flare ribbons. The C II integrated intensity (see Figure 1) is correlated with the RHESSI 12-25 keV light curve. The line intensity in selected flare points increased by several orders of magnitude without a significant increase of the line half-width, which may reflect nonthermal processes (e.g. nonthermal ionization and excitation of the atoms).

- The CII 1334.5 A line redshift formed a stable structure across the flare region. This structure did not change significantly during the flare revealing the presence of a background dynamical process. However, the integrated time curve of the redshift is correlated with the X-ray flux (see Figure 1). Possibly, this is due to a combination of active region downflows and a flare impact in the atmosphere.

- In the Fe XXI $1354.1 \AA$ line, we detected blueshifts of about $50 \mathrm{~km} / \mathrm{s}$. The temporal evolution of these blueshifts corresponding to hot plasma upflows is presented in Figure 2. Initially, they appear in two zones roughly coincided with the $12-25 \mathrm{keV}$ X-ray sources (Fig. 2d). After some time, the blueshifts fill the space between these zones (Fig. 2f). We interpret this phenomenon as "chromospheric evaporation" - an upflow of the chromospheric plasma heated to coronal temperatures by an energy release mechanism, and filling coronal loops.

- The X-ray spectrum obtained from RHESSI observations is well-approximated either by a single-temperature model with a nonthermal emission, or by a two-temperature pure thermal model.

\section{Discussion and Conclusion}

The chromospheric evaporation process is very important to study, because it allows us to understand flare energy release and transport mechanisms. Historically, two types of the chromospheric evaporations are distinguished: "gentle" evaporation characterized by 
subsonic upflows and relatively long time scales at about several minutes (see Antiochos \& Sturrock 1978, for details), and "explosive" evaporation with rapidly evolving supersonic velocities (e.g. Zarro and Lemen 1988). From the observational point of view, the gentle evaporation corresponds to blueshifts of both, the upper chromospheric and coronal lines. In the explosive evaporation, only the hot coronal lines are blueshifted (with the corresponding flow velocity of more than $\sim 200 \mathrm{~km} / \mathrm{s}$ ), but the chromospheric lines are redshifted (see works of Milligan et al. 2006; Milligan \& Dennis 2009, for observational examples).

It is hard to understand what type of evaporation occurs in the discussed event. The "cold" transition region line C II is redshifted during the flare, and the "hot" coronal line Fe XXI is blueshifted (see Figure 1). However, the evaporation process develops on the time scale of several minutes, and the evaporated plasma flow is definitely subsonic (about $50 \mathrm{~km} / \mathrm{s}$ ). Because of this, the evaporation process can be classified as "gentle". Theoretically, the gentle evaporation is predicted for the chromosphere heating by a heat flux from the energy release site or by a relatively low flux of high-energy electrons.

To understand the possible energy sources we calculated the energy which might be deposited by the injection of the accelerated electrons (the thermal-nonthermal fit to the X-ray spectrum): $P_{\text {nonth }}=\frac{\delta-1}{\delta-2} F\left(E>E_{\text {low }}\right) E_{\text {low }}=P_{\text {nonth }} \approx 5 \times 10^{27} \mathrm{erg} / \mathrm{s}$, and by the heat conduction (the two-temperature model fit): $Q_{\mathrm{cl}}=k_{0} T_{e}^{5 / 2} S \nabla T_{e} \sim$ $k_{0} T_{e}^{7 / 2} S / L \sim 10^{28} \mathrm{erg} / \mathrm{s}$ (Spitzer and Härm 1953). Surprisingly, we received approximately the same energy rates, which indicate that, perhaps, both processes of the energy transfer took place.

To summarize, we have analyzed the UV spectroscopic data for a M 1.0 class solar flare, and found several observational details. A strongly redshifted preflare jet and nonthermal emission at the beginning of the flare were observed. During the flare the "cold" lines of the transition region are redshifted, and the "hot" coronal Fe XXI line is blueshifted as predicted for "explosive" evaporation. However, the plasma flows are subsonic and develop on the time scale of minutes, corresponding to "gentle" evaporation. Thus, perhaps, this classification is ambiguous, and a larger statistical study is needed to understand the chromospheric evaporation properties in relation to different mechanisms of chromospheric heating caused by solar flares.

\section{References}

Antiochos, S. K. \& Sturrock, P. A. 1978, ApJ, 220, 1137

Bornmann, P. L., Speich, D., Hirman, J., et al. 1996, SPIE Conf. Ser., 2812, 291

Brosius, J. W. \& Phillips, K. J. H. 2004, ApJ, 613, 580

De Pontieu, B., Title, A. M., Lemen, J. R., et al. 2014, SoPh, 289,2733

Doschek, G. A., \& Warren, H. P., \& Young, P. R. 2013, ApJ, 767, 55

Goode, P. R., et al. 2010, Astronomische Nachrichten, 331, 620

Kumar, P., Yurchyshyn, V., Wang, H., \& Cho, K.-S. 2015, ApJ, 809, 83

Lemen, J. R., Title, A. M., Akin, D. J., et al. 2012, SoPh, 275, 17

Lin, R. P., Dennis, B. R., Hurford, G. J., et al. 2002, SoPh, 2010, 3

Milligan, R. O., Gallagher, P. T., Mathioudakis, M., \& Keenan, F. P., ApJL, 642, L169

Milligan, R. O. \& Dennis, B. R. 2009, ApJ, 699, 968

Milligan, R. O. 2015, ArXiv e-print, 1501.04829

Sadykov, V. M., et al. 2014, ArXiv e-print, 1412.0172v1

Sadykov, V. M., et al. 2015, ApJ, 805, 167

Scherrer, P. H., Schou, J., Bush, R. I., Kosovichev, A. G., et al. 2012, ApJ, 275, 207

Spitzer, L. \& Härm, R. 1953, Phys.Rev., 89, 977

Zarro, D. M. \& Lemen, J. R. 1988, ApJ, 329, 456 